\title{
Measurement of the branching fractions of $\Lambda_{c}^{+} \rightarrow p \bar{K} n(\pi)$
}

M. S. Alam, ${ }^{1}$ S. B. Athar, ${ }^{1}$ Z. Ling, ${ }^{1}$ A. H. Mahmood, ${ }^{1}$ H. Severini, ${ }^{1}$ S. Timm, ${ }^{1}$ F. Wappler, ${ }^{1}$ A. Anastassov, ${ }^{2}$ J. E. Duboscq, ${ }^{2}$ D. Fujino, ${ }^{2} *$ K. K. Gan ${ }^{2}$ T. Hart, ${ }^{2}$ K. Honscheid, ${ }^{2}$ H. Kagan, ${ }^{2}$ R. Kass, ${ }^{2}$ J. Lee, ${ }^{2}$ M. B. Spencer, ${ }^{2}$ M. Sung, ${ }^{2}$ A. Undrus, ${ }^{2, \dagger}$ R. Wanke, ${ }^{2}$ A. Wolf, ${ }^{2}$ M. M. Zoeller, ${ }^{2}$ B. Nemati, ${ }^{3}$ S. J. Richichi, ${ }^{3}$ W. R. Ross, ${ }^{3}$ P. Skubic, ${ }^{3}$ M. Bishai, ${ }^{4}$ J. Fast, ${ }^{4}$ J. W. Hinson, ${ }^{4}$ N. Menon, ${ }^{4}$ D. H. Miller, ${ }^{4}$ E. I. Shibata, ${ }^{4}$ I. P. J. Shipsey, ${ }^{4}$ M. Yurko, ${ }^{4}$ L. Gibbons, ${ }^{5}$ S. Glenn, ${ }^{5}$

S. D. Johnson, ${ }^{5}$ Y. Kwon ${ }^{5, \$}$ S. Roberts, ${ }^{5}$ E. H. Thorndike, ${ }^{5}$ C. P. Jessop, ${ }^{6}$ K. Lingel, ${ }^{6}$ H. Marsiske, ${ }^{6}$ M. L. Perl, ${ }^{6}$

D. Ugolini, ${ }^{6}$ R. Wang, ${ }^{6}$ X. Zhou, ${ }^{6}$ T. E. Coan, ${ }^{7}$ V. Fadeyev, ${ }^{7}$ I. Korolkov, ${ }^{7}$ Y. Maravin, ${ }^{7}$ I. Narsky, ${ }^{7}$ V. Shelkov, ${ }^{7}$ J. Staeck, ${ }^{7}$ R. Stroynowski, ${ }^{7}$ I. Volobouev, ${ }^{7}$ J. Ye, ${ }^{7}$ M. Artuso, ${ }^{8}$ A. Efimov, ${ }^{8}$ M. Goldberg, ${ }^{8}$ D. He,${ }^{8}$ S. Kopp, ${ }^{8}$ G. C. Moneti, ${ }^{8}$

R. Mountain, ${ }^{8}$ S. Schuh, ${ }^{8}$ T. Skwarnicki,${ }^{8}$ S. Stone,${ }^{8}$ G. Viehhauser, ${ }^{8}$ X. Xing, ${ }^{8}$ J. Bartelt, ${ }^{9}$ S. E. Csorna, ${ }^{9}$ V. Jain, ${ }^{9,8}$

K. W. McLean, ${ }^{9}$ S. Marka, ${ }^{9}$ R. Godang,${ }^{10}$ K. Kinoshita, ${ }^{10}$ I. C. Lai, ${ }^{10}$ P. Pomianowski, ${ }^{10}$ S. Schrenk,${ }^{10}$ G. Bonvicini ${ }^{11}$ D. Cinabro, ${ }^{11}$ R. Greene, ${ }^{11}$ L. P. Perera, ${ }^{11}$ G. J. Zhou ${ }^{11}$ B. Barish,${ }^{12}$ M. Chadha, ${ }^{12}$ S. Chan, ${ }^{12}$ G. Eigen, ${ }^{12}$ J. S. Miller, ${ }^{12}$

C. O'Grady, ${ }^{12}$ M. Schmidtler, ${ }^{12}$ J. Urheim, ${ }^{12}$ A. J. Weinstein, ${ }^{12}$ F. Würthwein, ${ }^{12}$ D. W. Bliss, ${ }^{13}$ G. Masek, ${ }^{13}$ H. P. Paar, ${ }^{13}$

S. Prell,${ }^{13}$ V. Sharma,${ }^{13}$ D. M. Asner, ${ }^{14}$ J. Gronberg, ${ }^{14}$ T. S. Hill, ${ }^{14}$ D. J. Lange, ${ }^{14}$ S. Menary,${ }^{14}$ R. J. Morrison, ${ }^{14}$

H. N. Nelson, ${ }^{14}$ T. K. Nelson, ${ }^{14}$ C. Qiao, ${ }^{14}$ J. D. Richman, ${ }^{14}$ D. Roberts, ${ }^{14}$ A. Ryd, ${ }^{14}$ M. S. Witherell,,${ }^{14}$ R. Balest, ${ }^{15}$

B. H. Behrens, ${ }^{15}$ W. T. Ford, ${ }^{15}$ H. Park, ${ }^{15}$ J. Roy, ${ }^{15}$ J. G. Smith, ${ }^{15}$ J. P. Alexander, ${ }^{16}$ C. Bebek, ${ }^{16}$ B. E. Berger ${ }^{16}$

K. Berkelman, ${ }^{16}$ K. Bloom, ${ }^{16}$ D. G. Cassel, ${ }^{16}$ H. A. Cho, ${ }^{16}$ D. S. Crowcroft, ${ }^{16}$ M. Dickson, ${ }^{16}$ P. S. Drell, ${ }^{16}$

K. M. Ecklund ${ }^{16}$ R. Ehrlich, ${ }^{16}$ A. D. Foland,${ }^{16}$ P. Gaidarev, ${ }^{16}$ B. Gittelman, ${ }^{16}$ S. W. Gray,${ }^{16}$ D. L. Hartill ${ }^{16}$ B. K. Heltsley, ${ }^{16}$ P. I. Hopman, ${ }^{16}$ J. Kandaswamy, ${ }^{16}$ P. C. Kim,${ }^{16}$ D. L. Kreinick, ${ }^{16}$ T. Lee, ${ }^{16}$ Y. Liu, ${ }^{16}$ G. S. Ludwig, ${ }^{16}$ N. B. Mistry, ${ }^{16}$

C. R. Ng, ${ }^{16}$ E. Nordberg, ${ }^{16}$ M. Ogg, ${ }^{16, \|}$ J. R. Patterson, ${ }^{16}$ D. Peterson, ${ }^{16}$ D. Riley, ${ }^{16}$ A. Soffer, ${ }^{16}$ B. Valant-Spaight,${ }^{16}$ C. Ward ${ }^{16}$ M. Athanas, ${ }^{17}$ P. Avery,${ }^{17}$ C. D. Jones, ${ }^{17}$ M. Lohner,${ }^{17}$ C. Prescott,,${ }^{17}$ J. Yelton, ${ }^{17}$ J. Zheng, ${ }^{17}$

G. Brandenburg, ${ }^{18}$ R. A. Briere, ${ }^{18}$ A. Ershov, ${ }^{18}$ Y. S. Gao, ${ }^{18}$ D. Y.-J. Kim, ${ }^{18}$ R. Wilson, ${ }^{18}$ H. Yamamoto, ${ }^{18}$ T. E. Browder, ${ }^{19}$ F. Li ${ }^{19}$ Y. Li,${ }^{19}$ J. L. Rodriguez,${ }^{19}$ T. Bergfeld ${ }^{20}$ B. I. Eisenstein, ${ }^{20}$ J. Ernst,${ }^{20}$ G. E. Gladding, ${ }^{20}$ G. D. Gollin, ${ }^{20}$

R. M. Hans ${ }^{20}$ E. Johnson, ${ }^{20}$ I. Karliner,${ }^{20}$ M. A. Marsh, ${ }^{20}$ M. Palmer,${ }^{20}$ M. Selen,${ }^{20}$ J. J. Thaler,${ }^{20}$ K. W. Edwards, ${ }^{21}$

A. Bellerive, ${ }^{22}$ R. Janicek, ${ }^{22}$ D. B. MacFarlane, ${ }^{22}$ P. M. Patel, ${ }^{22}$ A. J. Sadoff, ${ }^{23}$ R. Ammar, ${ }^{24}$ P. Baringer, ${ }^{24}$ A. Bean, ${ }^{24}$ D. Besson, ${ }^{24}$ D. Coppage, ${ }^{24}$ C. Darling, ${ }^{24}$ R. Davis, ${ }^{24}$ N. Hancock, ${ }^{24}$ S. Kotov,${ }^{24}$ I. Kravchenko, ${ }^{24}$ N. Kwak,${ }^{24}$

S. Anderson, ${ }^{25}$ Y. Kubota, ${ }^{25}$ S. J. Lee, ${ }^{25}$ J. J. O’Neill, ${ }^{25}$ S. Patton, ${ }^{25}$ R. Poling, ${ }^{25}$ T. Riehle, ${ }^{25}$ V. Savinov, ${ }^{25}$ and A. Smith ${ }^{25}$

(CLEO Collaboration)

\author{
${ }^{1}$ State University of New York at Albany, Albany, New York 12222 \\ ${ }^{2}$ Ohio State University, Columbus, Ohio 43210 \\ ${ }^{3}$ University of Oklahoma, Norman, Oklahoma 73019 \\ ${ }^{4}$ Purdue University, West Lafayette, Indiana 47907 \\ ${ }^{5}$ University of Rochester, Rochester, New York 14627 \\ ${ }^{6}$ Stanford Linear Accelerator Center, Stanford University, Stanford, California 94309 \\ ${ }^{7}$ Southern Methodist University, Dallas, Texas 75275 \\ ${ }^{8}$ Syracuse University, Syracuse, New York 13244 \\ ${ }^{9}$ Vanderbilt University, Nashville, Tennessee 37235 \\ ${ }^{10}$ Virginia Polytechnic Institute and State University, Blacksburg, Virginia 24061 \\ ${ }^{11}$ Wayne State University, Detroit, Michigan 48202 \\ ${ }^{12}$ California Institute of Technology, Pasadena, California 91125 \\ ${ }^{13}$ University of California, San Diego, La Jolla, California 92093 \\ ${ }^{14}$ University of California, Santa Barbara, California 93106 \\ ${ }^{15}$ University of Colorado, Boulder, Colorado 80309-0390 \\ ${ }^{16}$ Cornell University, Ithaca, New York 14853 \\ ${ }^{17}$ University of Florida, Gainesville, Florida 32611 \\ ${ }^{18}$ Harvard University, Cambridge, Massachusetts 02138 \\ ${ }^{19}$ University of Hawaii at Manoa, Honolulu, Hawaii 96822
}

\footnotetext{
*Permanent address: Lawrence Livermore National Laboratory, Livermore, CA 94551.

${ }^{\dagger}$ Permanent address: BINP, RU-630090 Novosibirsk, Russia.

${ }^{\ddagger}$ Permanent address: Yonsei University, Seoul 120-749, Korea.

${ }^{\S}$ Permanent address: Brookhaven National Laboratory, Upton, NY 11973.

"Permanent address: University of Texas, Austin TX 78712.
} 


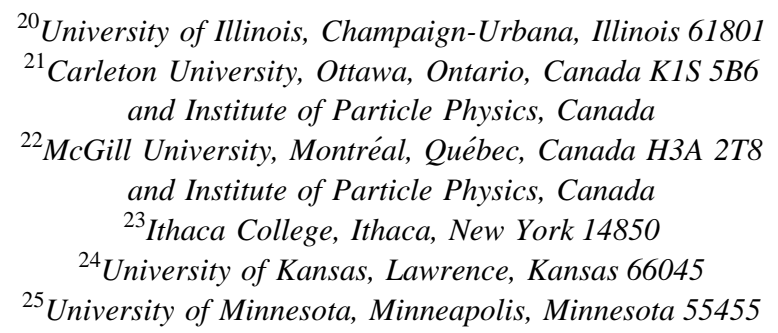

(Received 10 September 1997; published 18 February 1998)

Using data recorded by the CLEO-II detector at CESR, we report new measurements of the branching fractions for the decays of the charmed baryon $\Lambda_{c}^{+}$into $p K^{-} \pi^{+} \pi^{0}, p \bar{K}^{0}, p \bar{K}^{0} \pi^{+} \pi^{-}$, and $p \bar{K}^{0} \pi^{0}$, all measured relative to $p K^{-} \pi^{+}$. The relative branching fractions are $0.67 \pm 0.04 \pm 0.11,0.46 \pm 0.02 \pm 0.04,0.52 \pm 0.04 \pm 0.05$, and $0.66 \pm 0.05 \pm 0.07$, respectively.

[S0556-2821(98)05705-1]

PACS number(s): 14.20.Lq, 13.30.Eg, 14.65.Dw

Since the first observation of the lowest lying charmed baryon, the $\Lambda_{c}^{+}$, there have been many measurements made of its exclusive decay channels. As it is difficult to measure the production cross section of the $\Lambda_{c}^{+}$baryons, decay rates are typically presented as branching ratios relative to $\Lambda_{c}^{+}$ $\rightarrow p K^{-} \pi^{+}$, the most easily observed decay channel. However, fewer than half of the $\Lambda_{c}^{+}$hadronic decays are presently accounted for. Measurement of these modes is of practical as well as theoretical interest. Here, we present

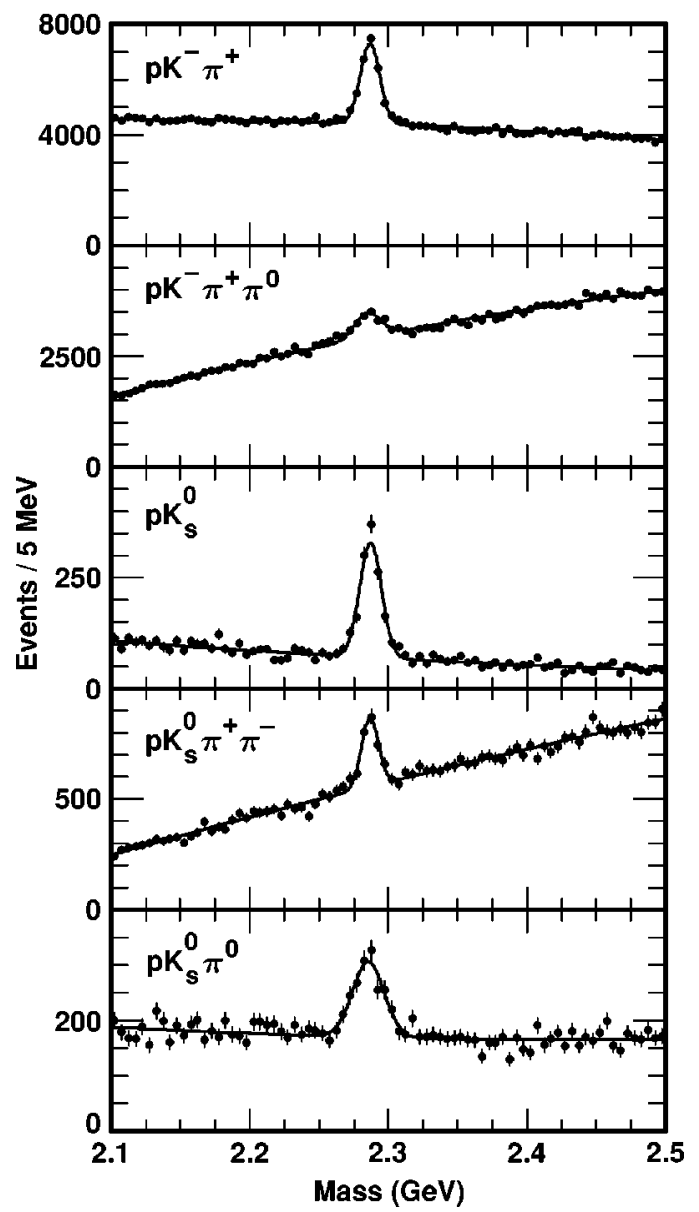

FIG. 1. Invariant mass plots for the 5 different decay modes of the $\Lambda_{c}^{+}$. measurements of the branching fractions of $\Lambda_{c}^{+}$into $p K^{-} \pi^{+} \pi^{0}, p \bar{K}^{0}, p \bar{K}^{0} \pi^{+} \pi^{-}$, and $p \bar{K}^{0} \pi^{0}$, all relative to $p K^{-} \pi^{+}$. The last of these is the first measurement of this mode. The other modes have been previously measured but with considerably larger uncertainties than in the present study.

The data presented here were taken by the CLEO II detector [1] operating at the Cornell Electron Storage Ring. The sample used in this analysis corresponds to an integrated luminosity of $4.8 \mathrm{fb}^{-1}$ from data taken on the $\Upsilon(4 S)$ resonance and in the continuum at energies just above and below the $Y(4 S)$. We detected charged tracks with a cylindrical drift chamber system inside a solenoidal magnet. Photons were detected using an electromagnetic calorimeter consisting of 7800 cesium iodide crystals.

Particle identification of $p, K^{-}$, and $\pi^{+}$candidates was performed using specific ionization measurements in the drift chamber and, when present, time-of-flight measurements. For each mass hypothesis, a combined $\chi^{2}$ probability $P_{i}$ was formed $(i=\pi, K, p)$. Using these probablilities, a normalized probability ratio $L_{i}$ was evaluated, where $L_{i}=P_{i} /\left(P_{\pi}+P_{K}+P_{p}\right)$. Well-identified protons peaked near $P_{p}=1.0$ while tracks that were identified to not be protons peak near $P_{p}=0.0$. For a track to be used as a proton in this study, we required it to have $L_{p}>0.8$, which eliminated much of the background, though with considerable diminution of efficiency. For kaons we applied a looser and more efficient cut of $L_{K}>0.1$. We have chosen these cuts using a Monte Carlo simulation program to maximize the significance of the signals. The proton identification requirement resulted in an efficiency that is strongly momentum dependent, being over $95 \%$ for momenta less than $1 \mathrm{GeV} / c$, fall-

TABLE I. The number of $\Lambda_{c}^{+}$, s found with $x_{p}\left(\Lambda_{c}\right)>0.5$.

\begin{tabular}{lcr}
\hline \hline Mode & MC width $(\mathrm{meV})$ & \multicolumn{1}{c}{ Signal } \\
\hline$p K^{-} \pi^{+}$ & 16 & $10109 \pm 191$ \\
$p K^{-} \pi^{+} \pi^{0}$ & 22 & $2606 \pm 165$ \\
$p \bar{K}^{0}$ & 19 & $1025 \pm 40$ \\
$p \bar{K}^{0} \pi^{+} \pi^{-}$ & 15 & $985 \pm 65$ \\
$p \bar{K}^{0} \pi_{0}$ & 27 & $774 \pm 52$ \\
\hline \hline
\end{tabular}


TABLE II. The measured relative branching fractions.

\begin{tabular}{lclr}
\hline \hline Mode & Relative efficiency & \multicolumn{1}{c}{$B / B\left(p K^{-} \pi^{+}\right)$} & Previous measurements \\
\hline$p K^{-} \pi^{+}$ & 1.0 & 1.0 & \\
$p K^{-} \pi^{+} \pi^{0}$ & 0.383 & $0.67 \pm 0.04 \pm 0.11$ & $0.72_{-0.22}^{+0.32} \quad[5]$ \\
$p \bar{K}^{0}$ & 0.218 & $0.46 \pm 0.02 \pm 0.04$ & $0.44 \pm 0.07 \pm 0.05[4]$ \\
& & & $0.55 \pm 0.17 \pm 0.14[6]$ \\
$p \bar{K}^{0} \pi^{+} \pi^{-}$ & 0.187 & $0.52 \pm 0.04 \pm 0.05$ & $0.62 \pm 0.15 \pm 0.03[7]$ \\
$p \bar{K}^{0} \pi^{0}$ & 0.115 & $0.66 \pm 0.05 \pm 0.07$ & $0.43 \pm 0.12 \pm 0.04[4]$ \\
\hline \hline
\end{tabular}

ing to $25 \%$ at $p=2 \mathrm{GeV} / c$. The kaon identification is rather efficient, with a mean value of $94 \%$ when averaged over all momenta. In order to reduce the large combinatoric background, we required $x_{p}>0.5$, where $x_{p}=P_{\Lambda_{c}} / \sqrt{E_{\text {beam }}^{2}-m_{\Lambda_{c}}^{2}}$ is the scaled momentum of the $\Lambda_{c}^{+}$candidate. Approximately $60 \%$ of $\Lambda_{c}^{+}$baryons from $c \bar{c}$ continuum events passed this requirement.

The $\bar{K}^{0}$ candidates were identified in their decay $K_{s}^{0} \rightarrow \pi^{+} \pi^{-}$, by reconstructing a secondary vertex from the intersection of two oppositely charged tracks in the $r-\phi$ plane. The secondary vertex was required to be displaced from the beam spot by at least $1 \mathrm{~mm}$ in the direction of flight of the $\overline{K^{0}}$, and each of the daughter particles was required to be inconsistent with coming from the beam spot. The invariant mass of the $\overline{K^{0}}$ candidate must lie within $9 \mathrm{MeV} / c^{2}$ [around 3 standard deviations $(\sigma)$ ] of its nominal value.

The $\pi^{0}$ candidates were selected through their decay $\pi^{0} \rightarrow \gamma \gamma$ from pairs of well-defined showers in the CsI calorimeter with a reconstructed invariant mass within $3 \sigma$ of the $\pi^{0}$ mass. In order to reduce the combinatorial background, each $\gamma$ was required to have an energy of at least $50 \mathrm{MeV}$, and the $\pi^{0}$ was required to have a momentum of at least $300 \mathrm{MeV} / c$.

The resulting mass distributions for the 5 modes are shown in Fig. 1. Each peak was fit to the sum of a Gaussian signal distribution with width fixed to that obtained from CLEO's GEANT based Monte Carlo simulation program and a second order polynomial background distribution. The signal widths used and the resulting signal yields are tabulated in Table I.

The efficiency for each $\Lambda_{c}^{+}$mode was calculated using the Monte Carlo simulation program [2]. The reconstruction efficiency of the $\Lambda_{c}^{+}$decays has some dependence on the resonant substructure of these states. In the case of the $p K^{-} \pi^{+}$mode, the Monte Carlo generator produced a mixture of non-resonant three-body decay together with $\Delta^{++} K^{-}$ and $p \bar{K}^{* 0}$ decays, according to their measured branching fractions [3]. These three types of decays had slightly different reconstruction efficiencies, so that including the substructure changes the efficiency by $\Delta \epsilon / \epsilon=0.02$ relative to 3 -body phase space. The difference in detection efficiency for $p K^{-} \pi^{+}$(non-resonant) compared with $p K^{-} \pi^{+}$resonating via $p \bar{K}^{* 0}$ was found to be $\approx 10 \%$. We have also investigated the dependence of the reconstruction efficiency of the other modes on a possible resonant substructure, including all modes involving an intermediate $K^{*}$. In no case did the substructure produce as large a change in efficiency as that noted above. The poor signal to background ratio did not allow a detailed measurement of the substructure. The efficiency calculation took into account the $\overline{K^{0}} \rightarrow K_{s}^{0}$ and $K_{s}^{0} \rightarrow \pi^{+} \pi^{-}$ branching fractions (see Table II).

We have considered many possible sources of systematic error in the measurement. The main contributors to the systematic uncertainty came from the following sources: first, uncertainties in the fitting procedures, which were estimated by looking at the changes in the yields using different orders of polynomial background and different signal widths $(15 \%$ in the case of $p K^{-} \pi^{+} \pi^{0}$, but much smaller for the other modes); second, uncertainties due to the unknown mix of resonant substructure in the multi-body decays (up to $3 \%$ depending on the mode); third, uncertainties due to $\pi^{0}$ finding $(5 \%)$, checked using the partial reconstruction of $D^{*+} \rightarrow D^{0} \pi^{+}, D^{0} \rightarrow K^{-} \pi^{+} \pi^{0}$ decays, the $K_{s}^{0}$ finding (5\%), found by comparing $K_{s}^{0}$ yields in the data and Monte Carlo simulation using a large variety of different algorithms, and track finding ( $1 \%$ per track), found by a detailed investigation of track parameters in $D^{*+} \rightarrow D^{0}, D^{0} \rightarrow K^{-} \pi^{+}$decays; and fourth, uncertainties in the reconstruction efficiency due to the particle identification criteria for protons and kaons (4\%), found by checking the agreement of Monte Carlo simulation of the energy-loss and time-of-flight measurements with the data from topologically identified $\Lambda \rightarrow p \pi^{-}$ and $D^{*+} \rightarrow K^{-} \pi^{+} \pi^{+}$decays.

These uncertainties have been added in quadrature to obtain the total systematic uncertainty for each mode. As the measurements are of ratios of branching fractions, many of the systematic uncertainties cancel.

There are three main types of quark decay diagrams that contribute to $\Lambda_{c}^{+}$decays. The simplest method is the simple spectator diagram in which the virtual $W^{+}$fragments independently of the spectator quark. The second method involves the quark daughters of the $W^{+}$combining with the remaining quarks. The third method, $W$ exchange, involves the $W^{+}$combining with the initial $d$ quark. Unfortunately all the decay modes under investigation here can proceed by more than one of these decay diagrams, and their decay rates are not amenable to calculation. Furthermore, our results are expressed as ratios of the branching fraction to that of $\Lambda_{c}^{+} \rightarrow p K^{-} \pi^{+}$, and there is no reliable measurement of the absolute branching fraction of this or any other exclusive decay mode, further hampering a comparison of experimental results and theory. 
In conclusion, we have measured new branching fractions of the $\Lambda_{c}^{+}$into 4 decay modes, measured relative to the normalizing mode $\Lambda_{c}^{+} \rightarrow p K^{-} \pi^{+}$. The results for three of these modes are in agreement with, and more accurate than, pre- vious measurements. We have made the first measurement of the decay rate of $\Lambda_{c}^{+} \rightarrow p \bar{K}^{0} \pi^{0}$. These measurements help account for the total width of the $\Lambda_{c}^{+}$and increase the understanding of charmed baryon decays.
[1] Y. Kubota et al., Nucl. Instrum. Methods Phys. Res. A 320, 66 (1992).

[2] R. Brun et al., Report No. CERN/DD/EE/84-11, 1984.

[3] Particle Data Group, R. Barnett et al., Phys. Rev. D 54, 1 (1996).
[4] P. Avery et al., Phys. Rev. D 43, 3599 (1991).

[5] S. Barlag et al., Z. Phys. C 48, 29 (1990).

[6] J. Anjos et al., Phys. Rev. D 41, 801 (1990).

[7] H. Albrecht et al., Phys. Lett. B 207, 109 (1988). 